\title{
Study on dynamic behavior analysis of towed line array sensor
}

\author{
Hyun Kyoung Shin ${ }^{1}$, Jung Soo Ryue ${ }^{1}$, Hyung-Taek $\mathrm{Ahn}^{1}$, Hee Seon $\mathrm{Seo}^{2}$ and Oh-Cho Kwon ${ }^{2}$ \\ ${ }^{I}$ School of Naval Architecture and Ocean Engineering, University of Ulsan, Ulsan, Republic of Korea \\ ${ }^{2} 6^{\text {th }}$ Research and Development Institute, Agency for Defense Development, Republic of Korea
}

\begin{abstract}
A set of equations of motion is derived for vibratory motions of an underwater cable connected to a moving vehicle at one end and with drogues at the other end. From the static analysis, cable configurations are obtained for different vehicle speeds and towing pretensions are determined by fluid resistance of drogues. Also the dynamic analysis is required to predict its vibratory motion. Nonlinear fluid drag forces greatly influence the dynamic tension. In this study, a numerical analysis program was developed to find out the characteristic of cable behaviour. The motion is described in terms of space and time coordinates based on Chebyshev polynomial expansions. For the spatial integration the collocation method is employed and the Newmark method is applied for the time integration. Dynamic tensions, displacements, velocities, accelerations were predicted in the time domain while natural frequencies and transfer functions were obtained in the frequency domain.
\end{abstract}

KEY WORDS: Underwater vehicle; Towed cable; Cable dynamics; Towed Array Sonar System (TASS).

\section{INTRODUCTION}

The cable used for marine applications includes mooring lines for ship berthing, deep sea mooring systems for floating offshore structures (Triantafyllou, et al., 1986) and tether cables for remotely operated vehicles (Grosenbaugh, 1991), etc.. Recently, underwater vehicles operate towed array sensor systems to expand detection range. This system can be regarded as a combined cable in about a thousand meters long (Hover, 1993). At this point, cable configuration and towing tension are very important for efficient operation. So, static and dynamic analyses are required to understand this system's behavior.

In this study, a nonlinear equation of motion was derived for cables (Shin, 1987). Nonlinear higher-order terms are eliminated to simplify the equations, however, important nonlinear terms such as nonlinear fluid drag and large dynamic tension were considered (Tse, et al., 1978; Shin, 1985). Numerical technique is used for the spectral analysis method which can obtain displacement, velocity and tension through time-domain analysis (Gottlieb and Orszag, 1977). Spectral analysis method is free from complicated processes such as determination of eigenmodes by using the orthogonal func-tions (Hildebrand, 1976) and can calculate the unknown variables accurately. The time-domain analysis has the advan-tages which do not need any nonlinearity equivalent replacement. It would rather use the Collocation method than the Galerkin method due to nonlinearity (Shin, 1987). Also, the Newmark's method was used for the time derivative.

\section{GOVERNING EQUATION}

Newton's second law can be written for an element with unstretched length $d s$ and stretched length $d p$ as

$$
m_{0} \frac{D v}{D t} d s=\sum_{i=0}^{n} \vec{F}_{i} d p
$$

Corresponding author: Hyun Kyoung Shin

e-mail:hkshin@ulsan.ac.kr 
where $m_{0}$ is mass per unit length in the unstretched coordinate, $\vec{v}=v_{1} \vec{t}+v_{2} \vec{n}+v_{3} \vec{b}$ is cable velocity, $s$ is unstretched distance of a material point from the origin, $\vec{F}_{i}$ is external force per unit stretched length of cable and $p$ is stretched distance of a same point from the origin.

The equation is rewritten as

$$
m_{0}\left[\frac{\partial \vec{v}}{\partial t}+\vec{w} \times \vec{v}\right]=\sum_{i=0}^{n} \vec{F}_{i}(1+e)
$$

Explicitly this is written as

$$
\begin{aligned}
& m_{0}\left[\frac{\partial v_{1}}{\partial t}-v_{2} w_{3}+v_{3} w_{2}\right]=\sum_{i=0}^{n} F_{t i}(1+e) \\
& m_{0}\left[\frac{\partial v_{2}}{\partial t}-v_{3} w_{1}+v_{1} w_{3}\right]=\sum_{i=0}^{n} F_{n i}(1+e) \\
& m_{0}\left[\frac{\partial v_{3}}{\partial t}-v_{1} w_{2}+v_{2} w_{1}\right]=\sum_{i=0}^{n} F_{b i}(1+e)
\end{aligned}
$$

Eq. (3) is the equation of motion for a cable expressed in the natural coordinate system. Subscript 1 is Tangential direction, subscript 2 is Normal direction. And subscript 3 is Bi-normal direction. Compatibility relation can be derived using displacements.

$$
\frac{D \vec{v}}{D s}=\frac{\partial e}{\partial t} \vec{t}+(1+e)(\vec{w} \times \vec{t})
$$

where $s$ is arc length coordinate. These are the compatibility relations in term of velocities. Explicitly this can be written as

$$
\begin{gathered}
\frac{\partial v_{1}}{\partial s}-v_{2} \Omega_{3}=\frac{\partial e}{\partial t} \\
\frac{\partial v_{2}}{\partial s}-v_{1} \Omega_{3}-v_{3} \Omega_{1}=(1+e) w_{3} \\
\frac{\partial v_{3}}{\partial s}+v_{2} \Omega_{1}=-(1+e) w_{2}
\end{gathered}
$$

Where $\Omega$ is Darboux vector.

The vertical unit vector in terms of the natural vector can be expressed.

$$
\vec{k}=\sin \phi \vec{t}+\cos \psi \cos \phi \vec{n}-\cos \phi \sin \psi \vec{b}
$$


The rotation vector expressed in Euler angles gives

$$
\vec{w} d t=(\sin \phi \delta \theta+\delta \psi) \vec{t}+(\cos \phi \cos \psi \delta \theta+\sin \psi \delta \phi) \vec{n}+(-\cos \phi \sin \psi \delta \theta+\cos \psi \delta \phi) \vec{b}
$$

The Darboux vector can be expressed in terms of Euler angles using Frenet's formulas (Hildebrand, 1976).

$$
\begin{gathered}
\frac{1}{\tau}=\frac{\partial \psi}{\partial s}-\tan \psi \tan \phi \frac{\partial \phi}{\partial s} \\
0=\frac{\partial \theta}{\partial s}+\frac{\partial \phi}{\partial s} \tan \psi \frac{1}{\cos \phi} \\
\frac{1}{\rho}=\frac{\partial \phi}{\partial s} \frac{1}{\cos \psi}
\end{gathered}
$$

The governing equations in terms of Euler angles are obtained.

$$
\begin{aligned}
& m_{0}\left[\frac{\partial v_{1}}{\partial t}+\left(\cos \phi \cos \psi \frac{\partial \theta}{\partial t}+\sin \psi \frac{\partial \phi}{\partial t}\right) v_{3}-\left(-\cos \phi \sin \psi \frac{\partial \theta}{\partial t}\right) v_{2}\right]=\frac{\partial T_{e}}{\partial s}+F_{t}(1+e)-w_{0} \sin \phi \\
& \left(m_{0}+m_{a_{0} n}\right) \frac{\partial v_{2}}{\partial t}-m_{0}\left(\sin \phi \frac{\partial \theta}{\partial t}+\frac{\partial \psi}{\partial t}\right) v_{3}+m_{0}\left(-\cos \phi \sin \psi \frac{\partial \theta}{\partial t}+\cos \psi \frac{\partial \phi}{\partial t}\right) v_{1} \\
& =\frac{\partial \phi}{\partial s} \frac{1}{\cos \psi} T_{e}+F_{n}(1+e)-w_{0} \cos \phi \cos \psi \\
& \left(m_{0}+m_{a_{0} b}\right) \frac{\partial v_{3}}{\partial t}+m_{0}\left(\sin \phi \frac{\partial \theta}{\partial t}+\frac{\partial \psi}{\partial t}\right) v_{2}-m_{0}\left(\cos \phi \cos \psi \frac{\partial \theta}{\partial t}+\sin \psi \frac{\partial \phi}{\partial t}\right) v_{1} \\
& =F_{b}(1+e)-w_{0} \cos \phi \sin \psi \\
& \frac{\partial \phi}{\partial s} \frac{1}{\cos \psi}=-\frac{\partial \theta}{\partial s} \frac{\cos \phi}{\sin \psi} \\
& \frac{\partial v_{1}}{\partial s}-\frac{v_{2}}{\cos \psi} \frac{\partial \phi}{\partial s}=\frac{\partial e}{\partial t} \\
& \frac{\partial v_{2}}{\partial s}-\left[\frac{\partial \psi}{\partial s}-\tan \psi \tan \phi \frac{\partial \phi}{\partial s}\right] v_{3}+\frac{v_{1}}{\cos \psi} \frac{\partial \phi}{\partial s}=(1+e)\left[-\cos \phi \sin \psi \frac{\partial \theta}{\partial t}+\cos \psi \frac{\partial \phi}{\partial t}\right] \\
& \frac{\partial v_{3}}{\partial s}-\left[\frac{\partial \psi}{\partial s}-\tan \psi \tan \phi \frac{\partial \phi}{\partial s}\right] v_{2}=-(1+e)\left[\cos \phi \cos \psi \frac{\partial \theta}{\partial t}+\sin \psi \frac{\partial \phi}{\partial t}\right] \\
& T_{e}=f(e)
\end{aligned}
$$


These constitute a set of eight equations with eight unknowns. Eq. (9) are the non-linear governing equations in terms of Euler angles.

\section{ANALYSIS MODEL}

The towed cable consists of three parts such as light weight cable(LWC), acoustic module(AM) and tail rope(TR) and disk drogue is attached at its end as shown in Fig. 1.

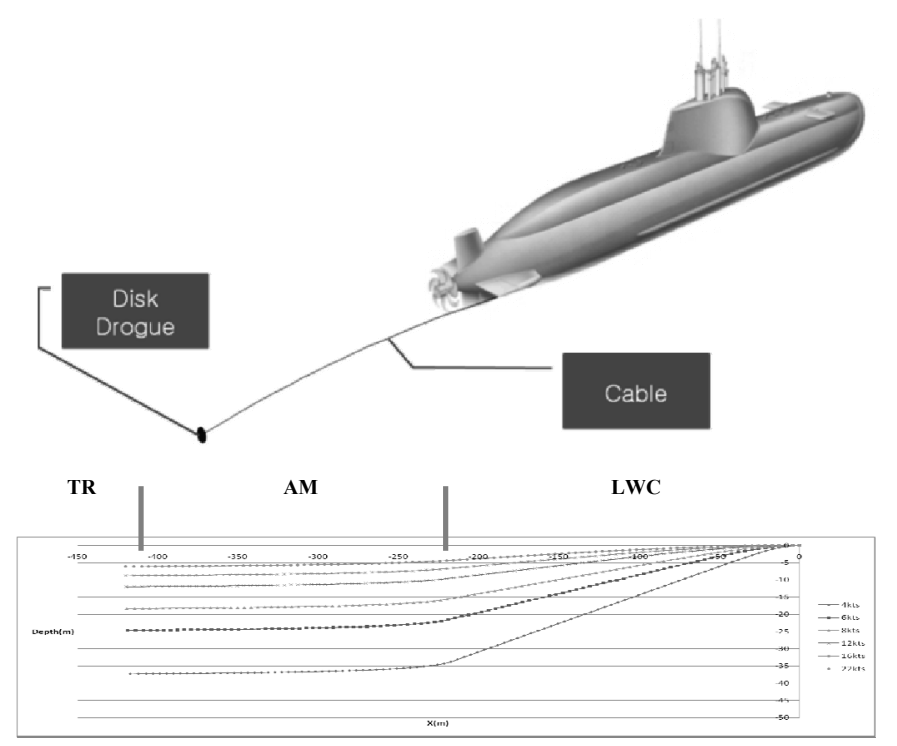

Fig. 1 Towed array sensor system.

The specification of the towed cable is shown in Tables $1 \sim 2$.

Table 1 Specifications of towed cable (1/2).

\begin{tabular}{|l|c|c|}
\hline Towed Cable & L.W.C & A.M \\
\hline Length $(m)$ & 195 & 195 \\
\hline Diameter $(m)$ & 0.03 & 0.06 \\
\hline Mass in air $(\mathrm{kg} / \mathrm{m})$ & 0.544 & 2.177 \\
\hline Added mass $(\mathrm{kg} / \mathrm{m})$ & 0.355 & 2.177 \\
\hline Weight in sea water $(N / m)$ & 1.852 & 0.000 \\
\hline $\operatorname{EA}(N)$ & 1523987 & 281392 \\
\hline
\end{tabular}

Table 2 Specifications of towed cable (2/2).

\begin{tabular}{|c|c|c|}
\hline Towed Cable & T.R & Disk Drogue \\
\hline Length $(m)$ & 10 & 0.1 \\
\hline Diameter $(m)$ & 0.03 & - \\
\hline Mass in air $(\mathrm{kg} / \mathrm{m})$ & 0.322 & - \\
\hline Added mass $(\mathrm{kg} / \mathrm{m})$ & 0.322 & - \\
\hline Weight in sea water $(\mathrm{N} / \mathrm{m})$ & 0.000 & - \\
\hline
\end{tabular}


LWC has a submerged weight in sea water. But AM and TR have neutral buoyancy in sea water.

\section{STATIC ANALYSIS}

In the static analysis, the drag coefficient of disk drogue is assumed to be 10, considering 10 disk drogues attached at the cable end. (Each disk drogue's drag coefficient is 1.) Cable configurations in different tow speeds are shown in Fig. 2. The vehicle tail is located at the origin $(0,0)$. With the increase in tow speed, the cable configuration becomes flatter because of the increase of cable tangential drag forces. Specially, TR remains tangential to the local flow incident on itself.

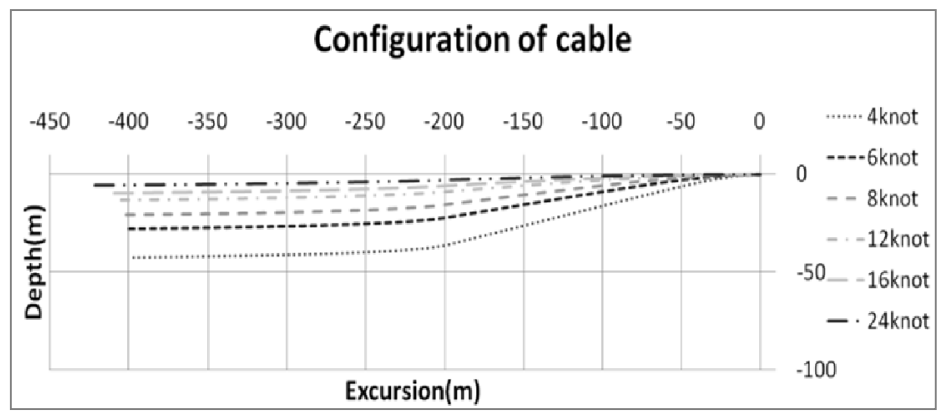

Fig. 2 Cable configurations in different tow speeds.

Fig. 3 shows cable tensions at different tow speeds. The increase in tow speed causes the increase in the drogue's drag and the tension along the towed cable. Therefore, the maximum tension can be expected at the vehicle's tail with the highest tow speed.

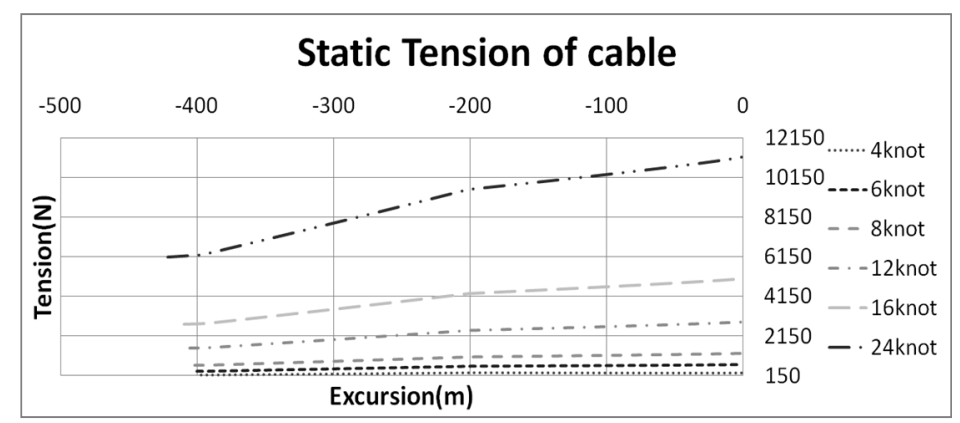

Fig. 3 Cable tensions in different tow speeds.

\section{DYNAMIC ANALYSIS}

The configuration of cable towed at 8 knots is shown in Figure 4. Its natural frequencies were calculated in Table 3.

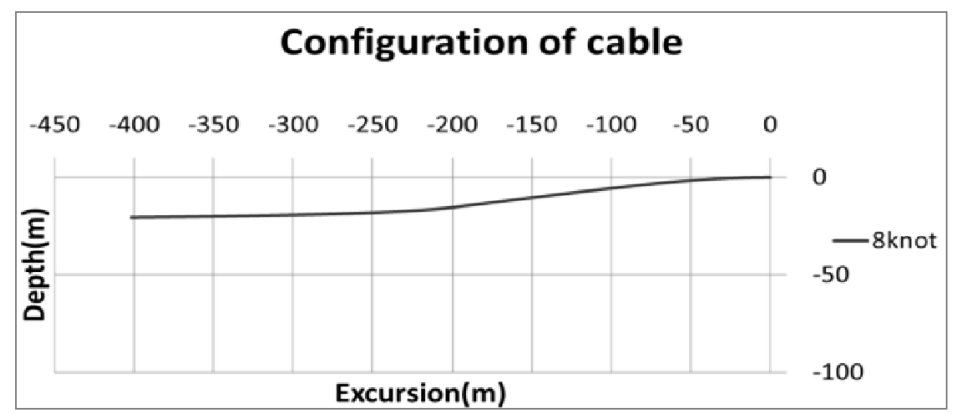

Fig. 4 TASS cable configuration (8knot). 
Table 3 Natural frequency of TASS (8knot).

\begin{tabular}{|c|c|}
\hline \multicolumn{2}{|c|}{ Natural frequency $(\mathrm{rad} / \mathrm{sec})$} \\
\hline 1st natural frequency & 0.1455469 \\
\hline 2nd natural frequency & 0.3226563 \\
\hline 3rd natural frequency & 0.4967968 \\
\hline 4th natural frequency & 0.6268747 \\
\hline 5th natural frequency & 0.7880463 \\
\hline 6th natural frequency & 0.9746867 \\
\hline 7th natural frequency & 1.1239837 \\
\hline
\end{tabular}

Also, dynamic tensions, displacements and transfer functions were obtained. The motion is described in terms of space and time coordinates based on Chebyshev polynomial expansions(Gottlieb and Orszag, 1977; Hildebrand, 1976). For the spatial integration the collocation method is employed and the Newmark method is applied for the time integration (Shin, 1987). The moving boundary condition and the fixed boundary condition were applied at the vehicle tail and at the disk drogue, respecttively, as shown in Fig. 5.

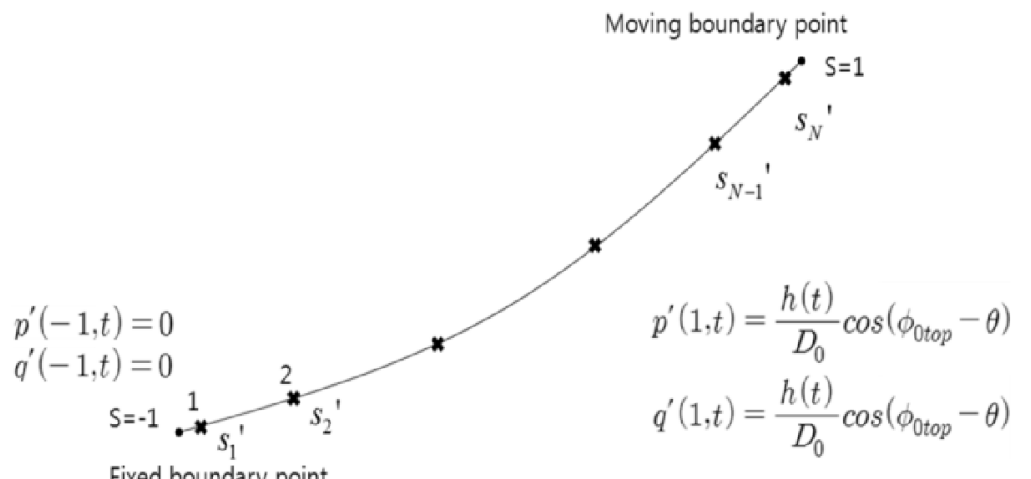

Fig. 5 Boundary conditions.

Location of collocation points were defined in Fig. 6, using Chebyshev polynomials. Especially, at the moving boundary point(11th), the acoustic module (6th) and the disk drogue(1st), displacements and tensions are calculated.

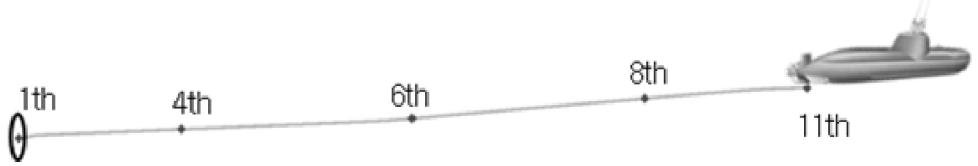

Fig. 6 TASS cable collocation points.

\section{Sinusoidal excitation}

The sine normal excitation with the amplitude of cable diameter and its first natural frequency $0.146 \mathrm{rad} / \mathrm{sec}$ in Table 3 was given at the $11^{\text {th }}$ collocation point of the cable towed at 8 knots in Figure 4. It appears in the form of the normal displacement nondimensionalized by cable diameter in Fig. 7. 


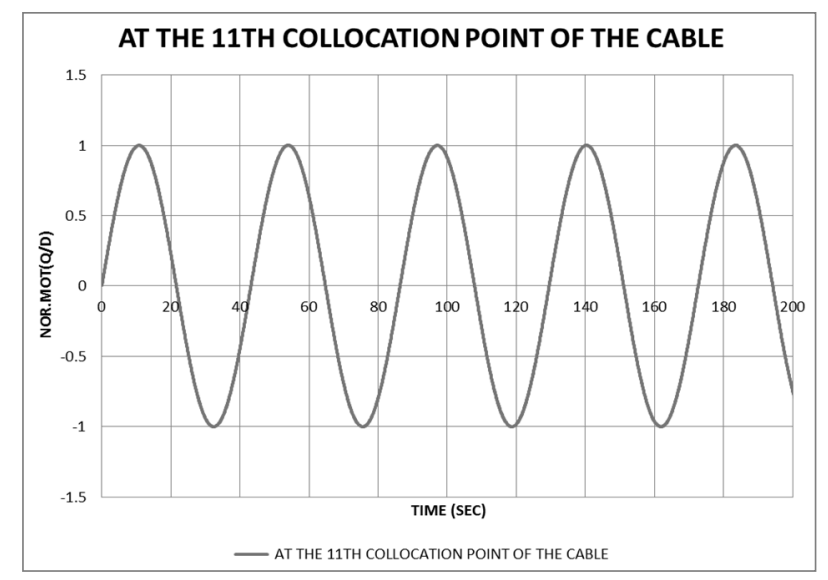

Fig. 7 Nondimensionalized normal displacement at the $11^{\text {th }}$ point (vehicle tail).

Normal drag coefficient is 1.0. Dynamic and static tensions at the vehicle tail(11th), are shown in Fig. 8. Dynamic tension is less than $1 \%$ of static tension.

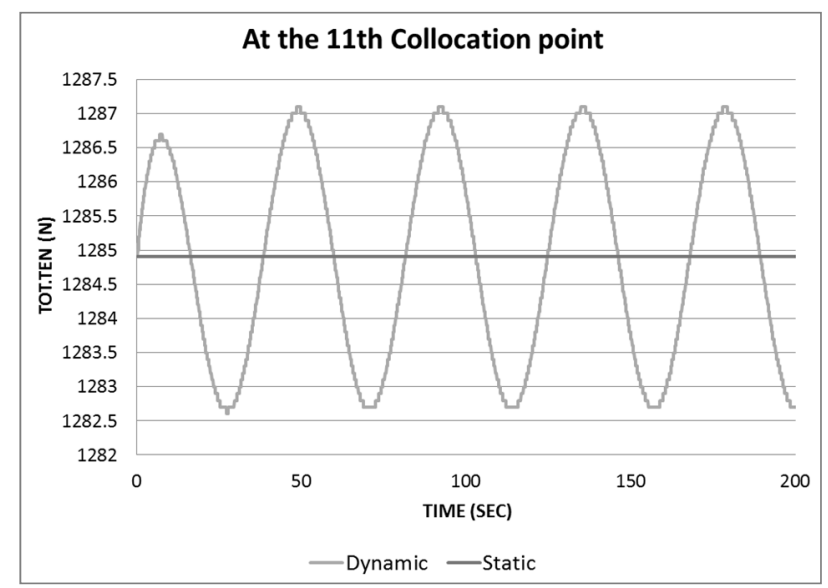

Fig. 8 Dynamic and static tension at vehicle tail.

In Fig. 9, dynamic and static tensions at acoustic module (6th) are shown.

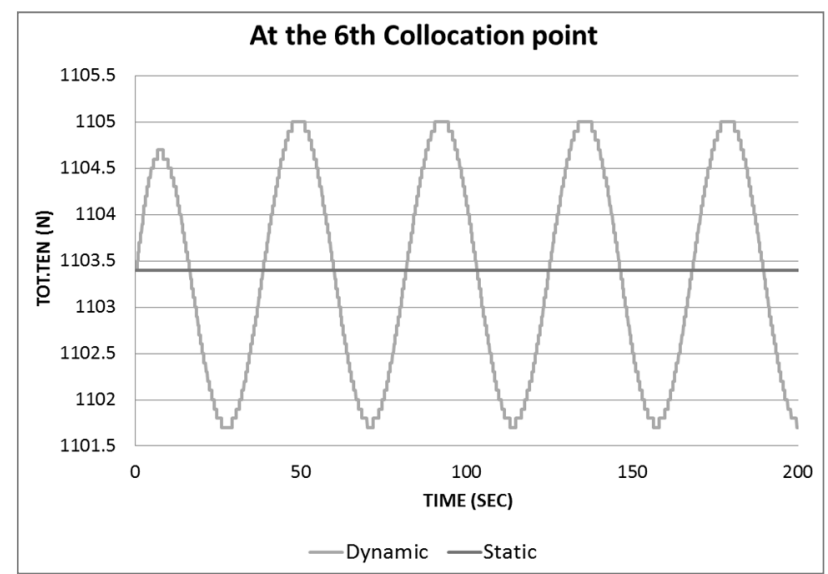

Fig. 9 Dynamic and static tension at acoustic module.

In Fig. 10, dynamic and static tensions at disk drogue(1st) are shown. 


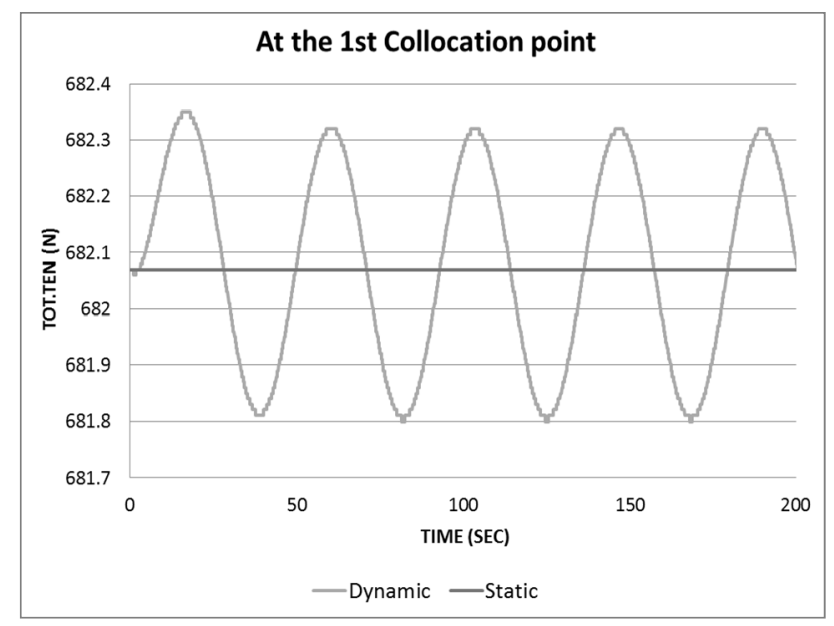

Fig. 10 Dynamic and static tension at disk drogue.

In the frequency domain, transfer functions were obtained. Figures 11, 12 and 13 show the ratio of the dynamic tension amplitude to the normal excitation amplitude at the frequency range of 0.1 to $1.0 \mathrm{rad} / \mathrm{sec}$.

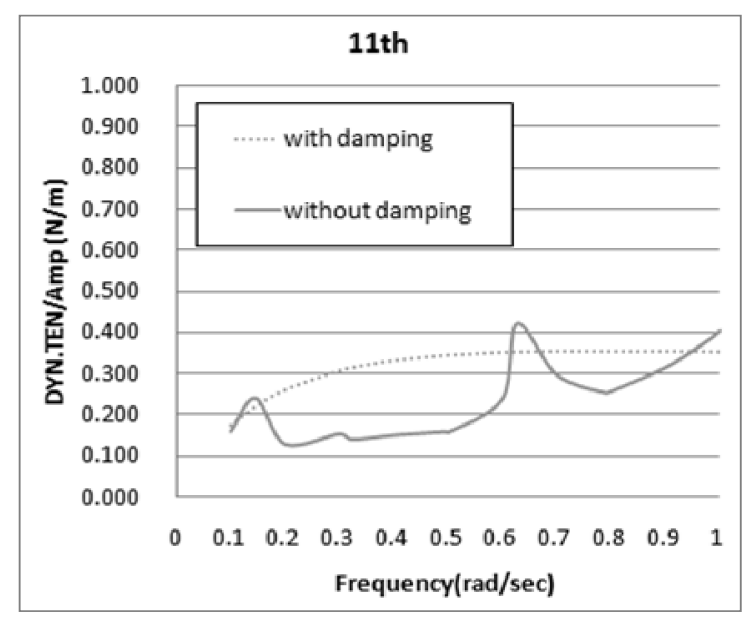

Fig. 11 Transfer function at vehicle tail.

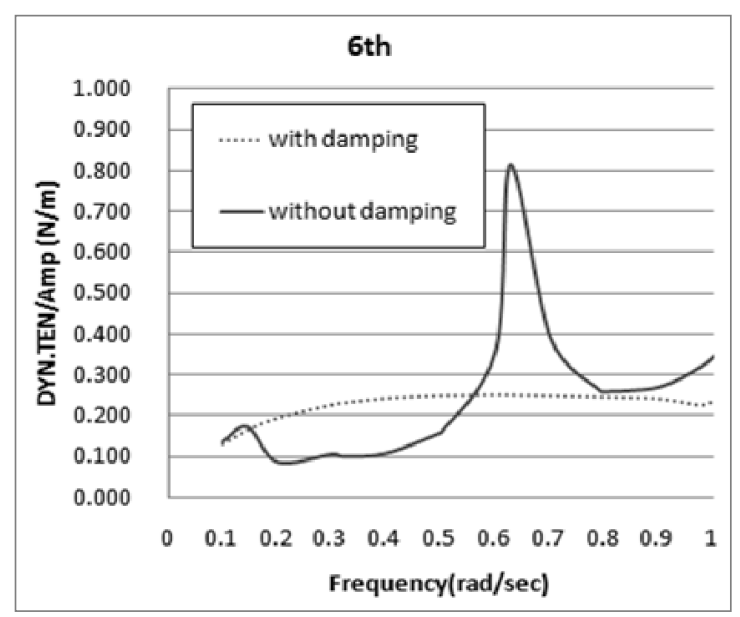

Fig. 12 Transfer function at acoustic module.

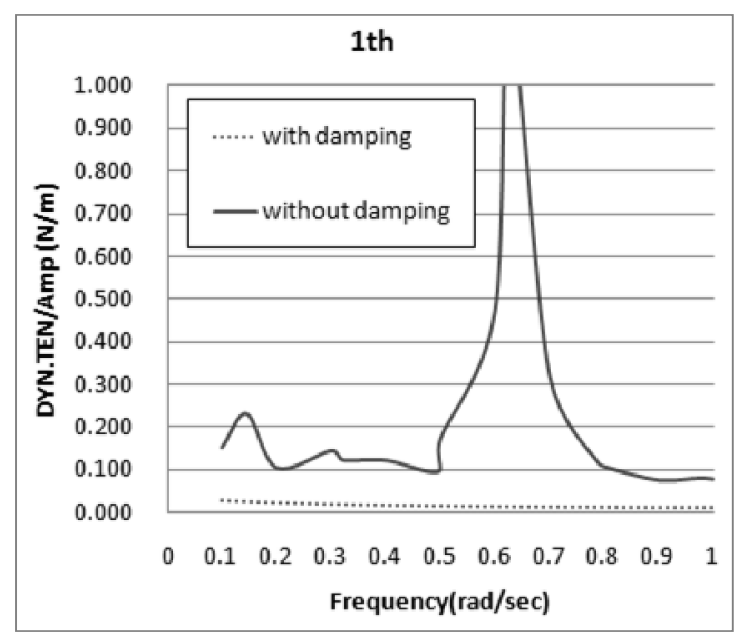

Fig. 13 Transfer function at disk drogue. 


\section{Irregular signal excitation}

Irregular normal excitation in Fig. 14 was applied to underwater vehicle tail(11th) and the displacement at the acoustic module (6th) was simulated.

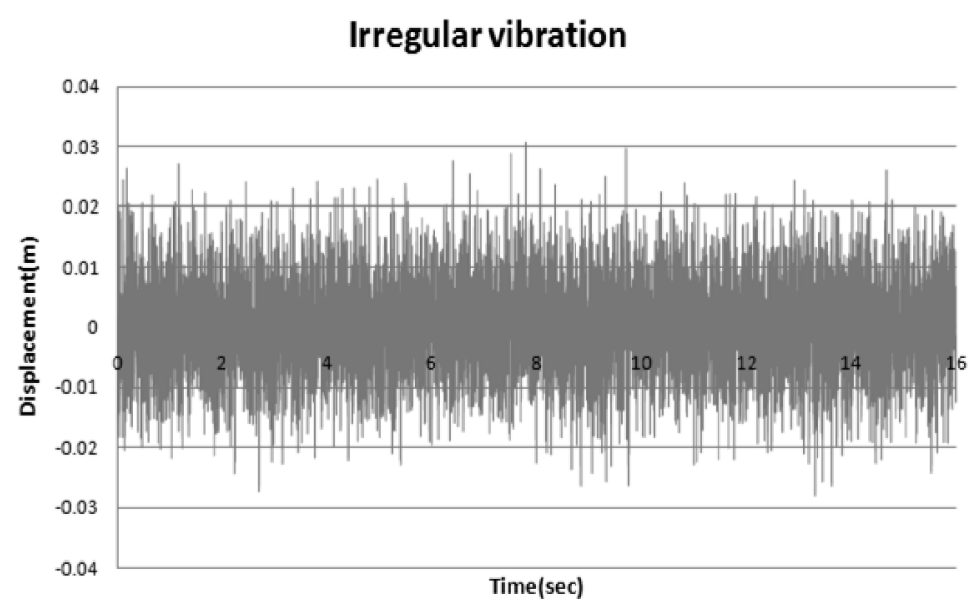

Fig. 14 Irregular normal excitation applied at the vehicle tail (11th).

The amplitude of excitation is the same as the cable diameter. Table 4 shows input time data for the exciation.

Table 4 Input time data.

\begin{tabular}{|l|l|}
\hline Time length & $16 \mathrm{sec}$ \\
\hline Sampling rate & $1024 \mathrm{~Hz}$ \\
\hline No. of data & 16384 \\
\hline Amplitude range of the input time signal & $-0.02 \sim 0.02$ \\
\hline Frequency range & $0 \sim 150 \mathrm{~Hz}$ \\
\hline
\end{tabular}

Fig. 15 shows the time history of dynamic tension simulated at the acoustic module. It is less than $0.1 \%$ of static tension. Fig. 16 shows the time history of normal displacement at the acoustic module(6th).

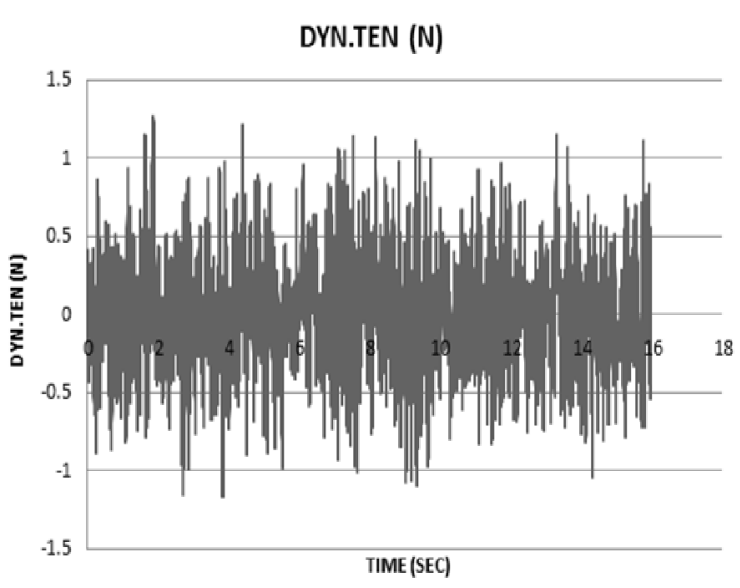

Fig. 15 Dynamic tension at acoustic module(6th).

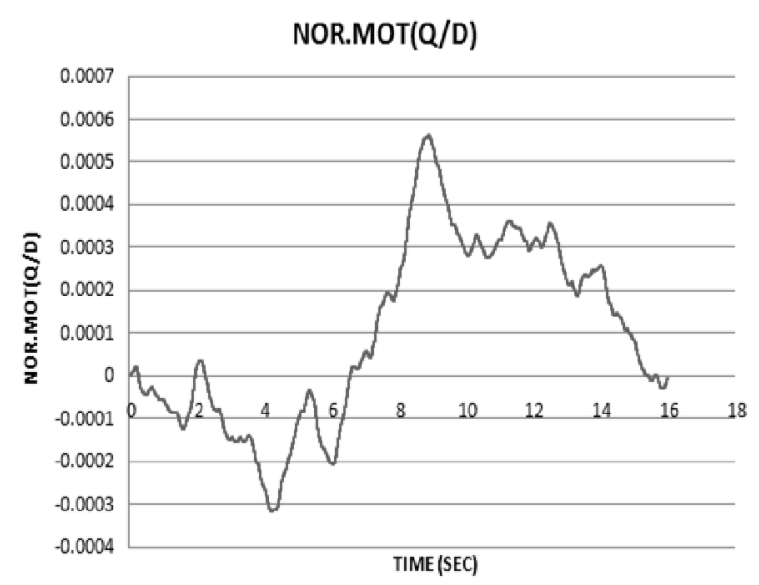

Fig. 16 Normal displacement at acoustic module. 
The spectrum of the normal displacement at the acoustic module is shown in Fig. 17 and peak frequency is $0.0625 \mathrm{~Hz}$.

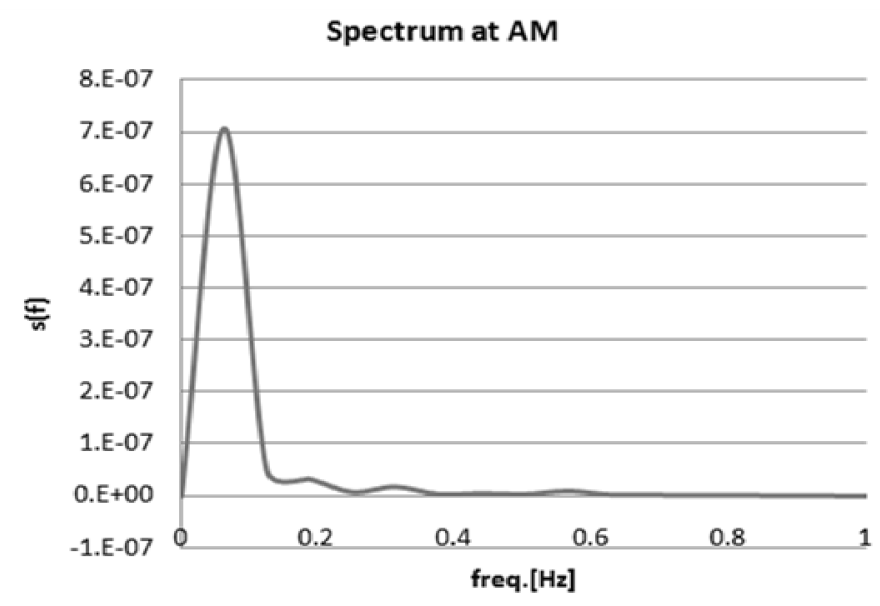

Fig. 17 Spectrum of normal displacement at the acoustic module.

\section{RESULTS AND DISCUSSIONS}

The static analysis of the towed cable with drogues at its end was performed and its configuration was obtained with static tensions along the cable. The increase in the tow speed causes the increase in the tension along the cable with the configuration flatter.

Also, the dynamic analysis was carried out when the tow speed is 8 knots. In the frequency domain, sinusoidal normal excitations were applied at the underwater vehicle tail and transfer functions were calculated at the acoustic module and the disk drogue. In the time domain analysis, the irregular normal excitation with the frequency range of $0 \sim 150 \mathrm{~Hz}$ was applied and the time history of dynamic tension and displacement were simulated at the acoustic module.

\section{CONCLUSIONS}

In the present study, a set of equations of motion was derived for static, dynamic and vibratory behaviors of a towed cable connected to a moving underwater vehicle at one end and with drogues at the other end. The cable consists of multisegments with different submerged weights in sea water.

From the static analysis, cable configurations were obtained for various towing speeds and towing pretensions were determined by fluid drag of drogues. As the tow speed increases, it is found that the pretensions increase and the cable configurations become very close to the shape flattened like a straight line.

Also the dynamic analysis is required to predict its dynamic and vibratory motions. Nonlinear fluid drag forces greatly influence the dynamic tension. In this study, a numerical analysis program based on the spectral method was developed to find out the characteristics of cable dynamic behaviour. Displacements, velocities, accelerations and total tensions including dynamic tensions along the cable were simulated in the time domain while natural frequencies and transfer functions were predicted in the frequency domain.

In the irregular normal signal excitation, the time history of dynamic tension and displacement at the acoustic module were analyzed for the vibratory motion behavior of the towed cable.

\section{ACKNOWLEDGEMENTS}

This research was funded by the $6^{\text {th }}$ Research and Development Institute, Agency for Defense Development, Republic of Korea (South). 


\section{REFERENCES}

Gottlieb, D. and Orszag, S.A., 1977. Numerical Analysis of Spectral Methods : Theory and Applications. Society for Industrial and applied mathematics.

Grosenbaugh, M.A., Yoerger, D.R. and Hover, F.S., 1991. Drag forces and flow induced vibrations of a long vertical tow cable. Journal of Offshore Mechanics and Arctic Engineering, 113.

Hildebrand, F.B., 1976. Advanced Calculus for Applications. 2nd Ed. Prentice Hall, Inc.

Hover, F.S., 1993. Methods for positioning deeply-towed underwater cables. Ph.D. thesis. Massachusetts Institute of Technology.

Shin, H., 1985. Numerical solution of the cable dynamic equations using the linearized equivalent damping force, Ocean Engineer thesis, Massachusetts Institute of Technology.

Shin, H., 1987. Nonlinear cable dynamics. Ph.D. thesis. Massachusetts Institute of Technology.

Triantafyllou, Michel S., Bliek, A., Burgess, J. and Shin, H., 1986. Sea grant college program, Mooring dynamics for offshore applications part1, MITSG 86-1.

Tse, F.S., Morse, I.E. and Hinkle, R.T., 1978. Mechanical Vibrations theory and applications. 2nd Ed. Allyn and Bacon, Inc., F.S. 